\title{
Differential expression of APE1 in hepatocellular carcinoma and the effects on proliferation and apoptosis of cancer cells
}

\author{
Zhipeng Sun, Yubing Zhu, Aminbuhe, Qing Fan, Jirun Peng*, Nengwei Zhang* \\ Oncology Surgery Department, Beijing Shijitan Hospital, Capital Medical University (Peking University Ninth School of Clinical \\ Medicine), Beijing, China.
}

\begin{abstract}
Summary
This research aimed to investigate the differential expression of apurinic-apyrimidinic endonuclease 1 (APE1) in hepatocellular carcinoma (HCC) tissues and cells and the effects on proliferation and apoptosis of cancer cells. Immunohistochemical techniques were used to detect the expression of APE1 in 80 cases of $\mathrm{HCC}$ and the corresponding paracancerous tissue microarrays; meanwhile, Western blots were used to detect the expression of APE1 in both human HCC BEL-7402, BEL-7405, HCC-9204, Hep3B, HepG2, SMMC-7721 and Huh7 cells, and normal hepatocyte L-02 cells. The relationship between APE1 expression and clinical pathological characteristics of HCC was statistically analyzed. APE1 shRNA vector was constructed in Hep $3 \mathrm{~B}$ cells to establish a stably transfected cell line, using Western blots to determine the interference efficiency. Cell proliferation activity was detected with MTT assays, while apoptosis was detected with the Annexin V-FITC/PI double-labeling technique. The expression of APE1 in HCC tissues and cells was significantly up-regulated, and its expression was significantly different from TNM staging and histopathological grading. Down-regulation of APE1 expression significantly reduced the proliferative activity and increased the apoptosis rate of Hep 3B cells. In conclusion, APE1 demonstrates cancer progression potential at the clinical, tissue and cell level. It provides a new idea and theoretical basis for APE1-based clinical diagnosis, prognosis determination and molecular targeted therapy in treatment of $\mathrm{HCC}$.
\end{abstract}

Keywords: APE1, hepatocellular carcinoma, differential expression, cell proliferation, apoptosis

\section{Introduction}

Hepatocellular carcinoma (HCC) is a type of primary liver cancer with high mortality clinically (1). As shown by the latest cancer statistics released by National Central Cancer Registry: In 2015, there were about 4.29 million new cases of malignant tumors and about 2.81 million deaths, which included about 461,100 new cases of HCC and 422,100 deaths (2), seriously threatening human life and health. Since uncontrolled growth, deterioration and metastasis were the main causes of high cancer mortality, the hotspots of HCC research in recent years have focused on exploring the molecular mechanisms of carcinogenesis and development of HCC, and searching

\footnotetext{
*Address correspondence to:

Dr. Jirun Peng and Dr. Nengwei Zhang, Oncology Surgery Department, Beijing Shijitan Hospital, Capital Medical University (Peking University Ninth School of Clinical Medicine), Beijing 100038, China.

E-mail: pengjr@medmail.com.cn or zhangnw1@sohu.com
}

for the biomarkers of malignant proliferation and interventional targets of HCC (3-5). Many studies have shown that apurinic-apyrimidinic endonuclease 1 (APE1) was expressed in a variety of tumors such as lung cancer, colorectal cancer, ovarian cancer and multiple myeloma (6), but in-depth study reports in HCC were very limited. This study was designed to investigate the expression characteristics of APE1 in HCC, analyze its relationship with clinical pathological characteristics, and determine its effects on HCC cell proliferation and apoptosis.

\section{Materials and Methods}

\subsection{Tissue samples and main reagents}

Tissue samples and experimental cells: HCC tissue microarrays were purchased from Shanghai Outdo Biotech (model HLivH160CS01); there were 80 cases of HCC, 1 each at cancer/paracancerous site. TNM staging was available, clinical phase 1, 2, 3 and 4, 
pathological grade I, II and III (AJCC, 7th Edition). The specific display arrangement is shown in Figure 1B. Both human HCC BEL-7402, BEL-7405, HCC9204, Hep3B, HepG2, SMMC-7721 and Huh-7 cells and normal hepatocyte L-02 cells APE1 were purchased from Institute of Basic Medical Sciences of Peking Union Medical College.

Main reagents: Total RNA extraction reagent TRIizol, SYBR ${ }^{\circledR}$ PrimeScript ${ }^{\mathrm{TM}}$ RT-PCR Kit and mRNA SYBR Green fluorescence quantification PCR reagent were purchased from Takara of Japan; protein extraction RIPA lysate, BCA protein concentration kit, SDS-PAGE gel preparation reagent, MTT cell proliferation kit and Annexin V-FITC/PI double-labeling apoptosis kit were purchased from Jiangsu Beyotime Biotechnology; cell transfection reagent Lipo 3000 was purchased from Invitrogen of the United States; APE1 antibody and GAPDH antibody were purchased from ABCAM of the United Kingdom.

\subsection{Methods}

Detection of APE1 expression level in HCC tissue microarrays with immunohistochemistry: After baking, hydration and antigen-repairing, the primary and secondary antibodies were added dropwise to tissue microarrays, followed by DAB color development and mounting with neutral balsam. After microphotography under a light microscope, IPP software was used to acquire integrated content density (IOD).

Detection of cellular APE1 expression with Western blots: The cells were collected to extract total proteins for quantification using the BCA technique. Following SDS-PAGE gel electrophoresis, the proteins were transferred to the membrane and blocked with 5\% skim milk powder-TBST blocking solution at room temperature for $1 \mathrm{~h}$. Then, diluted APE1 and GADPH primary antibodies were separately added to the $\mathrm{NC}$ membrane, which was incubated at $4^{\circ} \mathrm{C}$ overnight on a shaker; the corresponding secondary antibodies (1:100 dilution) were added, followed by incubation at $37^{\circ} \mathrm{C}$ constant temperature for $1 \mathrm{~h}$ on a shaker before color development.

Construction of APE1 shRNA vector: APE1 shRNA sequences were designed and synthesized, the forward primer was: 5'-CCGGCCTGGATTAAGAAGAAAGGA TCTCGAGATCCTTTCTTCTTAATCCAGGTTTTT-3', the reverse primer was: 5'-AATTCAAAAACCTGGAT TAAGAAGAAAGGATCTCGAGATCCTTTCTTCTT AATCCAGG-3', the clone was ligated to pLKO.1-GFP plasmid, followed by sequencing verification.

Detection of cell proliferation with MTT assay: The cells in each group were stably sub-cultured for passaging and then cultured in complete medium. MTT assay was conducted for $72 \mathrm{~h}$. Four duplicates were used in each group. A $20 \mu \mathrm{L}$ MTT (dissolved in 5mg/ mL PBS) was added to each well, and the supernatant was discarded after incubation for $4 \mathrm{~h}$. Then, $150 \mu \mathrm{L}$ Formazan solution was added to shake for $10 \mathrm{~min}$ until the crystals were fully dissolved. The absorbance was measured with a microplate reader $(570 \mathrm{~nm})$.

Detection of apoptosis with Annexin V-FITC/PI double-labeling technique: The cells were collected, placed on a 100 mesh copper sieve and rubbed gently. After rinsing with normal saline, the cells were centrifuged at $2500 \mathrm{r} / \mathrm{min}$, then the cell suspension was obtained after the supernatant and cell debris were discarded. Flow cytometry was employed for the analysis according to "Annexin V-FITC/PI Double-Labeling Detection Kit".

\subsection{Statistical processing}

SPSS 17.0 software was employed for statistical analysis, and $\overline{\mathrm{x}} \pm s$ was used for statistical description. One-way ANOVA was conducted to compare intergroup differences, if the results showed that the intergroup difference had statistical significance, $t$-test was further conducted for pairwise comparison of inter-group differences. All statistical analysis test levels were set at $\alpha=0.05$.

\section{Results}

\subsection{Differential expression of APE1 in HCC tissues}

Immunohistochemical technique was used to detect APE1 expression in HCC of the 80 cases and the corresponding paracancerous tissue microarrays; the IOD values are shown in Figure 1A. The results showed that APE1 expression was significantly up-regulated in HCC tissues (Figure 1C), and the difference had statistical significance $(p<0.0001)$. The results suggested that APE1 may play an important role in the development of HCC. The immunohistochemical results of 4 cases were selected from each group (Figure 2).

\subsection{Differential expression of APE1 in HCC cells}

Western blots were used to detect APE1 expression in human HCC BEL-7402, BEL-7405, HCC-9204, Hep3B, HepG2, SMMC-7721 and Huh-7 cells as well as in normal hepatocyte L-02 cells. The results showed that APE1 expression was higher in HCC cells than in normal hepatocytes to varying levels, APE1 expression in HCC cells was 2.5-3.6 times higher than in hepatocytes (Figure $3)$. The changes of differential expression of APE1 in HCC cells were consistent with that in HCC tissues, showing significant over-expression in both cases.

\subsection{Relationship between APE1 expression and pathological characteristics of HCC}

According to the statistical analysis of case data in 


\begin{tabular}{|c|c|c|c|c|c|c|c|c|c|c|c|c|c|c|c|c|}
\hline & 1 & 2 & 3 & 4 & 5 & 6 & 7 & 8 & 9 & 10 & 11 & 12 & 13 & 14 & 15 & 16 \\
\hline A & 217 & 194 & 206 & 178 & 493 & 261 & 817 & 322 & 1043 & 709 & 637 & 978 & 1406 & 534 & 412 & 169 \\
\hline B & 289 & 133 & 109 & 275 & 219 & 288 & 306 & 291 & 940 & 741 & 796 & 577 & 3951 & 406 & 1824 & 557 \\
\hline C & 263 & 460 & 398 & 304 & 308 & 628 & 435 & 378 & 1227 & 735 & 1406 & 532 & 2904 & 444 & 1399 & 384 \\
\hline D & 553 & 350 & 484 & 303 & 176 & 342 & 510 & 336 & 1667 & 430 & 1174 & 608 & 241 & 756 & 1103 & 281 \\
\hline E & 160 & 190 & 365 & 230 & 255 & 498 & 480 & 232 & 761 & 724 & 258 & 802 & 991 & 738 & 715 & 1030 \\
\hline $\mathrm{F}$ & 182 & 243 & 335 & 301 & 178 & 279 & 156 & 234 & 2388 & 733 & 1006 & 144 & 1803 & 556 & 1057 & 661 \\
\hline$G$ & 203 & 317 & 443 & 325 & 483 & 311 & 202 & 649 & 2023 & 749 & 226 & 704 & 2508 & 255 & 2990 & 537 \\
\hline $\mathrm{H}$ & 119 & 281 & 254 & 201 & 280 & 566 & 372 & 714 & 1013 & 223 & 1996 & 274 & 192 & 271 & 831 & 962 \\
\hline I & 154 & 230 & 282 & 357 & 316 & 400 & 249 & 263 & 1456 & 287 & 543 & 255 & 2377 & 206 & 1750 & 212 \\
\hline $\mathrm{J}$ & 137 & 266 & 237 & 209 & 180 & 287 & 860 & 441 & 460 & 852 & 203 & 304 & 1462 & 778 & 1405 & 574 \\
\hline
\end{tabular}

B

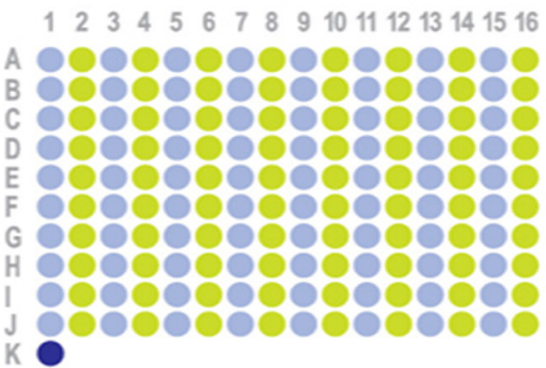

C

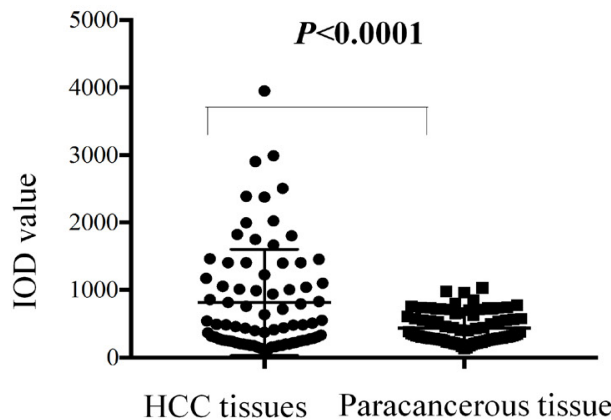

Figure 1. Differential expression of APE1 in HCC tissues. (A): IOD for APE1 expression in HCC of the 80 cases and the corresponding paracancerous tissue microarrays as detected with immunohistochemistry (Blue indicates HCC tissues, green indicates paracancerous tissues). (B): Schematic diagram for the distribution of HCC and paracancerous tissues in the microarrays (blue indicates HCC tissues, green indicates paracancerous tissues). (C): Statistical analysis of APE1 expression in HCC tissues.
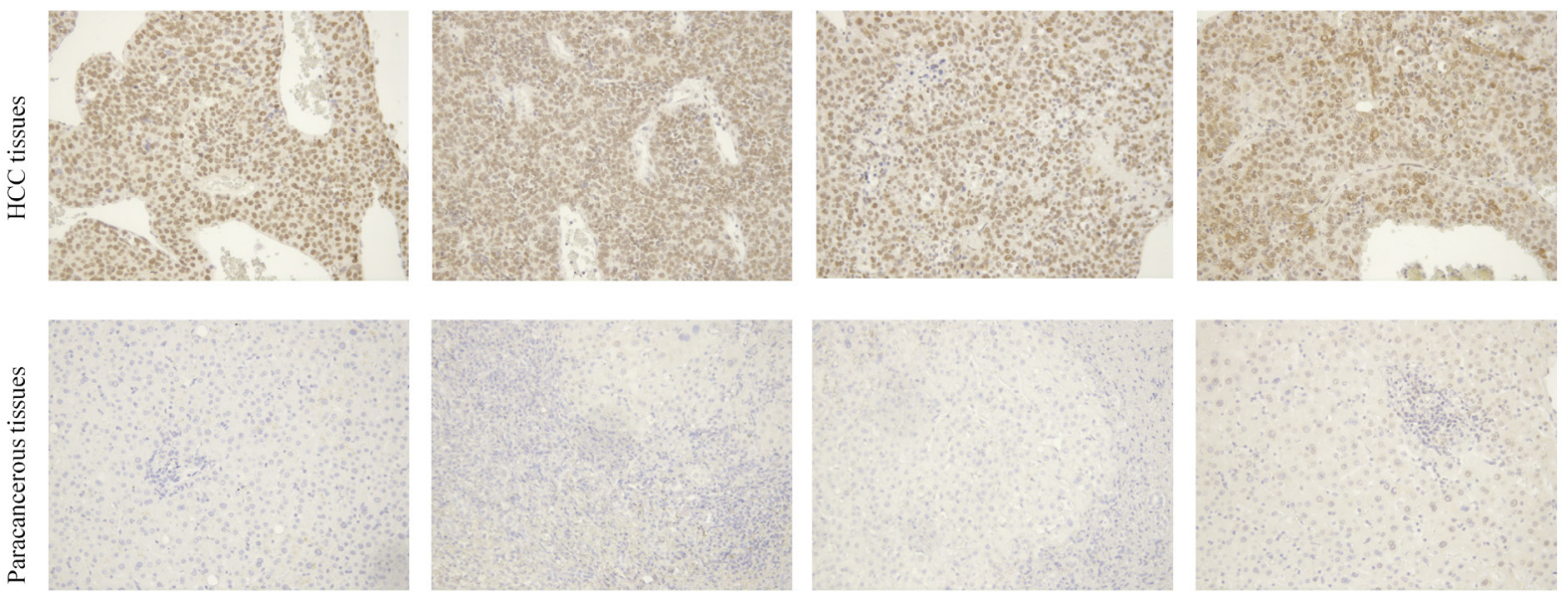

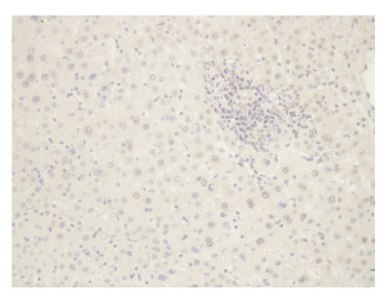

Figure 2. The immunohistochemical results of 4 cases were selected from HCC and paracancerous tissues in the microarrays. The immunohistochemistry results showed that the APE1 positive rate of HCC was much higher than paracancerous tissues in the microarrays. It was the same with the IOD result.

tissue microarrays, APE1 expression in HCC tissues of the 80 cases had no statistically significant correlation with patient's gender and age $(p>0.05)$, but its correlation with the differences in TNM staging and histological grading had statistical significance $(p<$ 0.05) (Table 1).

\subsection{Silencing APE1 expression and transfection efficiency evaluation}

The silencing of APE1 mRNA expression in HCC Hep 3B cells was detected with real-time PCR. The results showed that the mRNA expression level was significantly lower in APE1 shRNA group than in control shRNA group and blank group $(\mathrm{p}<0.05)$, and there was no significant difference between control shRNA group and blank group ( $p>0.05$ ) (Figure 4A). Meanwhile, protein expression detected with Western blotting was consistent with mRNA level, and the protein expression was significantly lower in APE1 shRNA group than in control shRNA group and blank group, demonstrating the efficacy of the designed shRNA interfering sequence (Figure 4B). Various groups of Hep $3 \mathrm{~B}$ cell lines with stably transfected plasmids were established, the transfection efficiency as detected by fluorescence microscopy was $>70 \%$, accordingly, the cell lines could be used for subsequent detections (Figure 4C). 


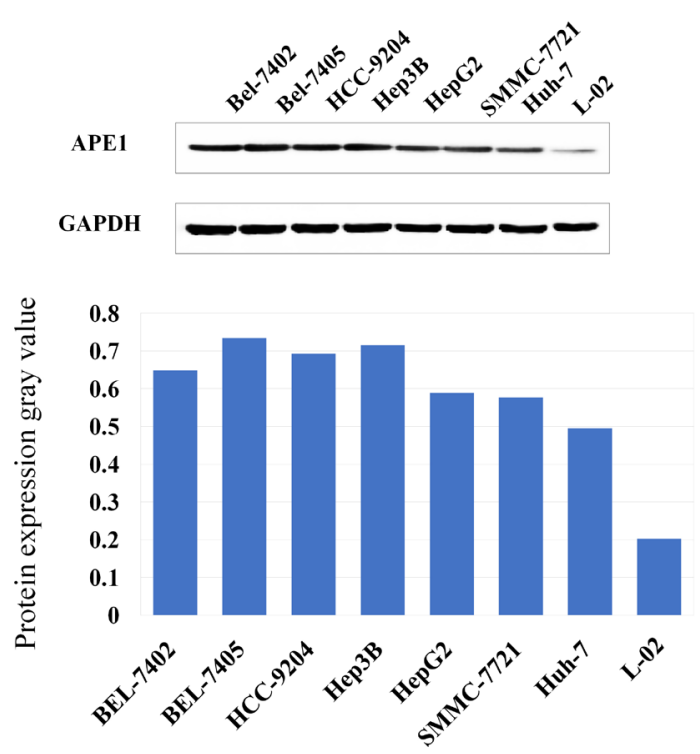

Figure 3. Detection of APE1 expression in human HCC cells with Western blot. APE1 expression in human HCC BEL7402, BEL-7405, HCC-9204, Hep3B, HepG2, SMMC-7721 and Huh- 7 cells as well as in normal hepatocyte L-02 cells. The results showed that APE1 expression was higher in HCC cells than in normal hepatocytes to varying levels, APE1 expression in HCC cells was 2.5-3.6 times higher than in hepatocytes.

\subsection{Effects of silencing APE1 on Hep $3 B$ cell proliferation ability}

MTT assay was used to detect cell proliferation in each group after culture for $72 \mathrm{~h}$. The results showed that the cell absorbance in blank group, control shRNA group and APE1 shRNA group was $1.525 \pm 0.079,1.497 \pm$ 0.072 and $0.981 \pm 0.063$, respectively, based on the results of one-way ANOVA, the inter-group differences had statistical significance $(F=109.7, p<0.001)$. The cell proliferation activity was significantly lower in APE1 shRNA group than in control shRNA group, and the difference had statistical significance $(p<0.001)$, while the difference in cell proliferation activity between blank group and control shRNA group had no statistical significance $(p>0.05)$. The results showed that silencing APE1 expression could significantly reduce cell proliferation activity in HCC Hep 3B cells (Figure 5).

\subsection{Effects of silencing APE1 expression on HCC Hep 3 B apoptosis}

An Annexin V-FITC/PI double-labeling technique was

Table 1. Relationship between APE1 expression and clinical pathological characteristics of HCC

\begin{tabular}{|c|c|c|c|c|c|}
\hline Group & No. of cases $(n=80)$ & Positive expression $(n=31)$ & Negative expression $(n=49)$ & $\mathrm{c}^{2}$ value & $p$ value \\
\hline \multicolumn{6}{|l|}{ Sex } \\
\hline Male & 64 & 25 & 39 & 0.013 & 0.908 \\
\hline Female & 16 & 6 & 10 & & \\
\hline \multicolumn{6}{|l|}{ Age } \\
\hline$\leq 50$ years & 20 & 7 & 13 & 0.158 & 0.691 \\
\hline$>50$ years & 60 & 24 & 36 & & \\
\hline \multicolumn{6}{|l|}{ TNM staging } \\
\hline I-II & 39 & 10 & 29 & 5.975 & 0.014 \\
\hline III-IV & 41 & 21 & 19 & & \\
\hline \multicolumn{6}{|c|}{ Histopathological grading } \\
\hline Good (I) & 2 & 0 & 2 & 7.167 & 0.027 \\
\hline Medium (II, III) & 67 & 23 & 44 & & \\
\hline Poor (IV) & 11 & 8 & 3 & & \\
\hline
\end{tabular}

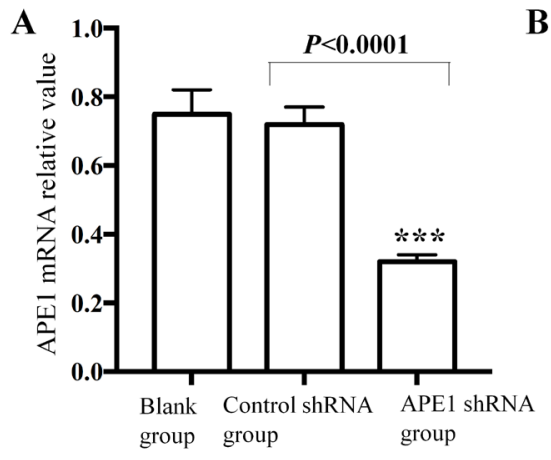

B

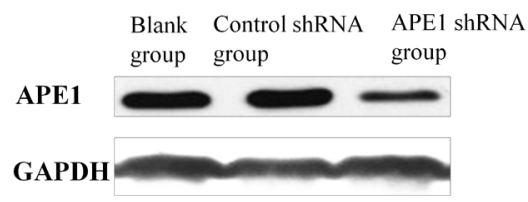

C

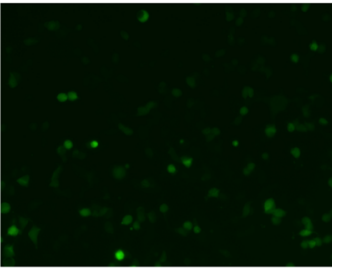

Control shRNA group

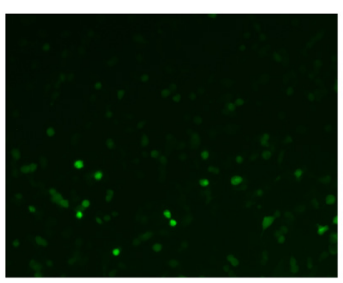

APE1 shRNA group

Figure 4. Silencing APE1 expression in HCC Hep 3B cells and transfection efficiency evaluation. (A): The mRNA expression level was significantly lower in APE1 shRNA group than in control shRNA group and blank group $(p<0.05)$, and there was no significant difference between control shRNA group and blank group $(p>0.05)$. (B): The APE1 protein expression was significantly lower in APE1 shRNA group than in control shRNA group and blank group. (C): The transfection efficiency as detected by fluorescence microscopy was $>70 \%$ in both experimental and control group. 
used to detect apoptosis in each group. The results showed that the total apoptosis rate in blank group, control shRNA group and APE1 shRNA group was $9.91 \% \pm 0.61 \%, 10.96 \% \pm 0.75 \%$ and $20.06 \% \pm 1.73 \%$, respectively. Based on one-way ANOVA, the intergroup differences had statistical significance $(F=46.63$, $p<0.01)$. The apoptosis rate was significantly higher in APE1 shRNA group than in control shRNA group, and the difference had statistical significance $(p<0.01)$; the difference in apoptosis rate between blank group and control shRNA group had no statistical significance ( $p$ $>0.05$ ) (Figure 6). The results showed that silencing APE1 expression was able to significantly increase the apoptosis of HCC Hep 3B, suggesting that APE1 may promote tumor growth by inhibiting apoptosis.

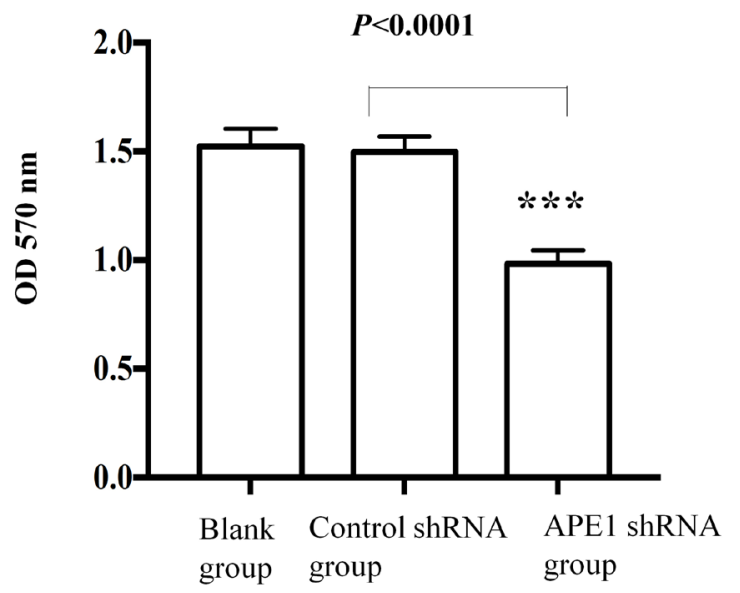

Figure 5. Effects of silencing APE1 expression on HCC Hep 3B cell proliferation ability. MTT assay was used to detect cell proliferation in each group after culture for $72 \mathrm{~h}$. The results showed that the cell absorbance in blank group, control shRNA group and APE1 shRNA group was $1.525 \pm 0.079,1.497$ \pm 0.072 and $0.981 \pm 0.063$, respectively, based on the results of one-way ANOVA, the inter-group differences had statistical significance $(F=109.7, p<0.0001)$.

\section{Discussion}

The carcinogenesis and development of HCC is a complex multi-factor multi-stage pathogenesis process that involves abnormal expression of many related genes (7). An important part of tumor biology is to identify the key genes of HCC and search for new therapeutic targets based on the study of mechanisms of action (8). APE1 is a protein that plays a role in DNA repair and redox function, and is able to regulate a variety of transcription factors associated with cancer-related pathways $(9,10)$. Its open reading frame consisting of 954bp encodes 318 amino acid residues (Figure 7) for protein diagram (11). On the one hand, APE1 functions as an endonuclease involved in DNA base excision repair, it is closely related to tumor cell proliferation; on the other hand, it regulates the activity of redox-sensitive transcription factors (12). Many studies showed APE1 over-expression in a variety of tumors (13-15). Some scholars used enzyme-linked immunosorbent assay to detect serum APE1 in patients suffering from bladder cancer without chemotherapy or radiotherapy (51 cases) and in a non-tumor control group ( 55 cases). The correlation between clinical factors and serum APE1 was determined by the area of subject's characteristics under the curve. The results showed significant over-expression of serum APE1 in patients with bladder cancer, the sensitivity and specificity were $93 \%$ and $59 \%$, respectively, and serum APE1 expression was related to tumor staging, grading, myometrial invasion and recurrence, suggesting that serum APE1 may serve as a potential serum marker of bladder cancer (16). Some studies also found that serum APE1 level was significantly elevated in 229 of 412 patients with non-small cell lung cancer and was correlated with tissue level $\left(r^{2}=0.639, p<0.001\right)$. Elevation of APE1 level in patient's tissues and serum prior to chemotherapy was related to progression-

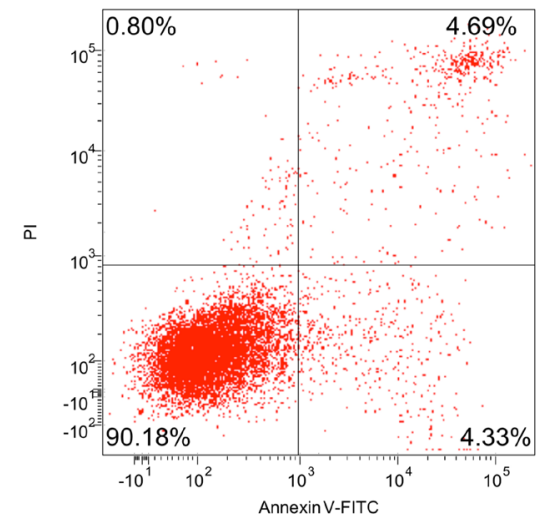

Blank group

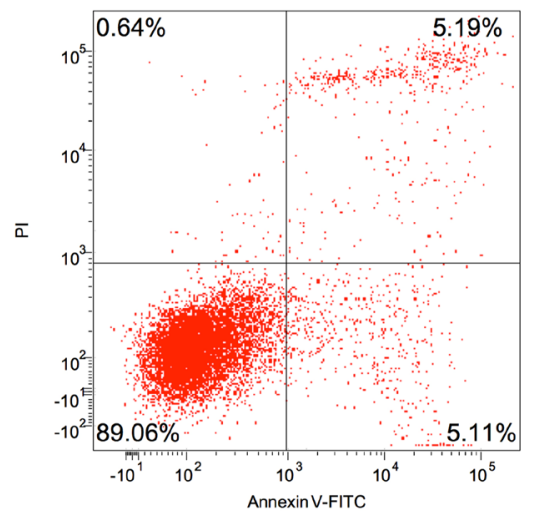

Control shRNA group

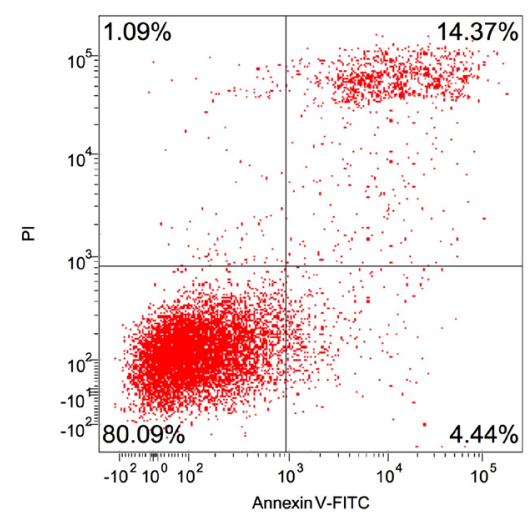

APE1 shRNA group

Figure 6. Silencing APE1 expression significantly increases HCC Hep 3B apoptosis. Annexin V-FITC/PI double-labeling technique was used to detect apoptosis in each group. The results showed that the total apoptosis rate in blank group, control shRNA group and APE1 shRNA group was $9.91 \% \pm 0.61 \%, 10.96 \% \pm 0.75 \%$ and $20.06 \% \pm 1.73 \%$, respectively. 


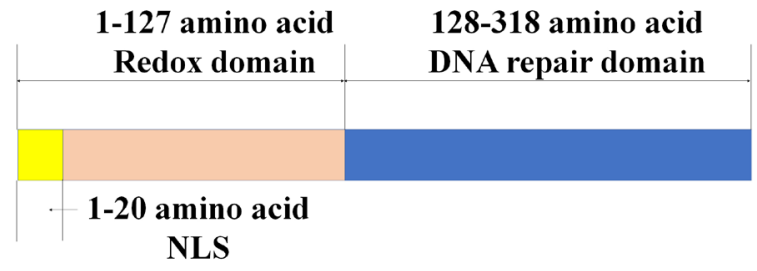

Figure 7. APE1 protein schematic diagram. The open reading frame of APE1 consisted of 954bp encoding 318 amino acid residues. The Redox domain, DNA repair domain are shown in this picture. So APE1 functions as an endonuclease involved in DNA base excision repair; on the other hand, it regulates the activity of redox-sensitive transcription factors.

free survival (HR: 2.165, $p<0.001$, HR: $1.421, p=$ 0.012 ), but unrelated to overall survival; after 6 cycles of chemotherapy, low APE1 serum level was related to better overall survival (HR: $0.497, p=0.010)(17)$.

With the rapid development of molecular biology technologies, some new methods such as shRNA technique are used in targeted intervention of APE1. RNA interference is a typical technique that uses double-stranded RNA to specifically inhibit gene expression of the corresponding complementary bases. This technique characterized by high specificity, small molecularity and transitivity can specifically silence gene expression. It has been widely used to explore gene functions and provide gene therapy for the treatment of malignant tumors (18). Bhat AA et al. knocked out APE1 with shRNA to study the pathogenesis of esophageal adenocarcinoma; they found that APE1 was essential for phosphorylation, nuclear localization and transcriptional activation of STAT3, further suggesting that APE1 plays a key role in inducing the activation of the EGFR-STAT3 signaling axis. It also responded to acidic bile salts and is a major risk factor for esophageal adenocarcinoma (19). Shah F et al. knocked out APE1 with siRNA in pancreatic cancer cells and found that some new genes and pathways affected APE1 expression using a single-cell sequencing technique, they also determined the specificity of tumor subtypes; these findings will facilitate hypothesis-driven methods to generate combination therapies. For example, pancreatic and other cancers can be treated with APE1 inhibitor APX33 30 in combination with other drugs approved by the FDA (20).

Since there were few study reports on the roles of APE1 in HCC pathogenesis, this study investigated its functions in human $\mathrm{HCC}$ high metastatic cells by silencing APE1. First of all, we examined the level of APE1 expression in 80 cases of HCC and the corresponding paracancerous tissue microarrays using immunohistochemical techniques. Meanwhile, we detected APE1 expression in human HCC BEL-7402, BEL-7405, HCC-9204, Hep3B, HepG2, SMMC-7721 and Huh-7 cells as well as in normal hepatocyte L-02 cells using Western blotting. The results showed that
APE1 expression in HCC was significantly up-regulated and different from TNM staging and histological grading. An APE 1 shRNA vector was further constructed in HCC Hep3B cells to establish stably transfected cell lines. Then, cell proliferative activity was detected with MTT assay while apoptosis was detected with an Annexin V-FITC/PI double-labeling technique. The results showed that down-regulation of APE1 expression significantly reduced the proliferative activity of Hep3B cells and increased the apoptosis rate. Apoptosis is not only an important means in the regulation of cell growth and differentiation but also an important protective mechanism against the tumors. The apoptotic potential is usually extremely low in cancerous cells, but the proliferative ability is enhanced, which directly leads to rapid pathologic division and proliferation of tumor cells, suggesting that APE1 may promote tumor growth by inhibiting apoptosis.

In summary, this experiment applied a RNA interference technique and successfully introduced an APE1 siRNA expression plasmid into human HCC cells, leading to down-regulation of cellular APE1 expression. It is confirmed that down-regulation of APE1 protein can inhibit proliferation and promote apoptosis of HCC cells. These findings provide an experimental basis and show certain prospects for clinical application of APE1 in the treatment of HCC, justifying further in-depth research.

\section{Acknowledgements}

This study was supported by China Railway Corporation science and technology development project, No.: J2017Z609 and the fund of Beijing Key Lab of Therapeutic Cancer Vaccines, No.: 2017-KF02. We thank these foundations.

\section{References}

1. Clark T, Maximin S, Meier J, Pokharel S, Bhargava P. Hepatocellular Carcinoma: Review of Epidemiology, Screening, Imaging Diagnosis, Response Assessment, and Treatment. Curr Probl Diagn Radiol. 2015; 44:479-486.

2. Chen W, Zheng R, Baade PD, Zhang S, Zeng H, Bray F, Jemal A, Yu XQ, He J. Cancer statistics in China, 2015. CA Cancer J Clin. 2016; 66:115-132.

3. Gao XH, Zhang SS, Chen H, Wang K, Xie W, Wang FB. Lipoprotein (a): A promising prognostic biomarker in patients with hepatocellular carcinoma after curative resection. Onco Targets Ther. 2018; 11:5917-5924.

4. Xiao H, Zhou B, Jiang N, Cai Y, Liu X, Shi Z, Li M, Du C. The potential value of CDV3 in the prognosis evaluation in Hepatocellular carcinoma. Genes Dis. 2018; 5:167-171.

5. Amr KS, Elmawgoud Atia HA, Elazeem Elbnhawy RA, Ezzat WM. Early diagnostic evaluation of miR-122 and miR-224 as biomarkers for hepatocellular carcinoma. Genes Dis. 2017; 4:215-221.

6. Li Q, Wei X, Zhou ZW, Wang SN, Jin H, Chen KJ, Luo J, Westover KD, Wang JM, Wang D, Xu CX, Shan 
JL. GADD45alpha sensitizes cervical cancer cells to radiotherapy via increasing cytoplasmic APE1 level. Cell Death Dis. 2018; 9:524.

7. Singh AK, Kumar R, Pandey AK. Hepatocellular Carcinoma: Causes, Mechanism of Progression and Biomarkers. Curr Chem Genom Transl Med. 2018; 12:926.

8. Lim, II, Farber BA, LaQuaglia MP. Advances in fibrolamellar hepatocellular carcinoma: A review. Eur J Pediatr Surg. 2014; 24:461-466.

9. Tell G, Quadrifoglio F, Tiribelli C, Kelley MR. The many functions of APE1/Ref-1: Not only a DNA repair enzyme. Antioxid Redox Signal. 2009; 11:601-620.

10. Fairlamb MS, Whitaker AM, Freudenthal BD. Apurinic/ apyrimidinic (AP) endonuclease 1 processing of AP sites with 5' mismatches. Acta Crystallogr D Struct Biol. 2018; 74:760-768.

11. Guerreiro PS, Estacio SG, Antunes F, Fernandes AS, Pinheiro PF, Costa JG, Castro M, Miranda JP, Guedes RC, Oliveira NG. Structure-based virtual screening toward the discovery of novel inhibitors of the DNA repair activity of the human apurinic/apyrimidinic endonuclease 1. Chem Biol Drug Des. 2016; 88:915-925.

12. Fung H, Demple B. A vital role for Ape1/Ref1 protein in repairing spontaneous DNA damage in human cells. Mol Cell. 2005; 17:463-470.

13. Logsdon DP, Shah F, Carta F, Supuran CT, Kamocka M, Jacobsen MH, Sandusky GE, Kelley MR, Fishel ML. Blocking HIF signaling via novel inhibitors of CA9 and APE1/Ref-1 dramatically affects pancreatic cancer cell survival. Sci Rep. 2018; 8:13759.

14. Hudson AL, Parker NR, Khong P, Parkinson JF, Dwight
T, Ikin RJ, Zhu Y, Chen J, Wheeler HR, Howell VM. Glioblastoma Recurrence Correlates With Increased APE1 and Polarization Toward an Immuno-Suppressive Microenvironment. Front Oncol. 2018; 8:314.

15. Yang X, Peng Y, Jiang X, et al. The regulatory role of APE1 in epithelial-to-mesenchymal transition and in determining EGFR-TKI responsiveness in non-small-cell lung cancer. Cancer Med. 2018; 7:4406-4419.

16. Shin JH, Choi S, Lee YR, Park MS, Na YG, Irani K, Lee SD, Park JB, Kim JM, Lim JS, Jeon BH. APE1/Ref-1 as a Serological Biomarker for the Detection of Bladder Cancer. Cancer Res Treat. 2015; 47:823-833.

17. Zhang $\mathrm{S}, \mathrm{He} \mathrm{L}$, Dai N, et al. Serum APE1 as a predictive marker for platinum-based chemotherapy of non-small cell lung cancer patients. Oncotarget. 2016; 7:7748277494.

18. Xin Y, Huang M, Guo WW, Huang Q, Zhang LZ, Jiang G. Nano-based delivery of RNAi in cancer therapy. Mol Cancer. 2017; 16:134.

19. Bhat AA, Lu H, Soutto M, Capobianco A, Rai P, Zaika A, El-Rifai W. Exposure of Barrett's and esophageal adenocarcinoma cells to bile acids activates EGFRSTAT3 signaling axis via induction of APE1. Oncogene. 2018.

20. Shah F, Goossens E, Atallah NM, Grimard M, Kelley MR, Fishel ML. APE1/Ref-1 knockdown in pancreatic ductal adenocarcinoma - characterizing gene expression changes and identifying novel pathways using single-cell RNA sequencing. Mol Oncol. 2017; 11:1711-1732.

(Received September 27, 2018; Revised October 18, 2018; Accepted October 21, 2018) 